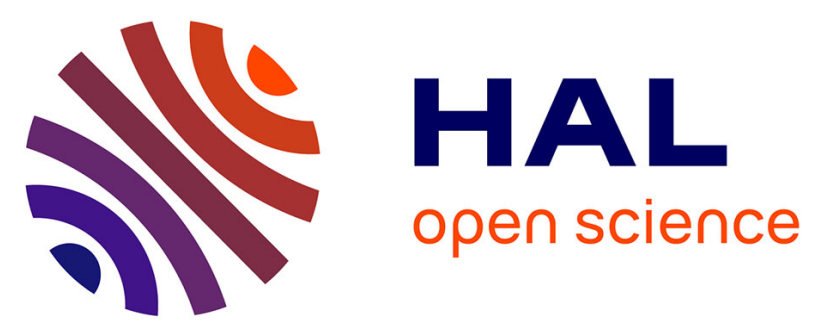

\title{
Permanences et mutations dans les terroirs rizicoles de Basse-Casamance (Sénégal)
}

Tidiane Sané, Catherine Mering, Marie-Christine Cormier-Salem, Ibrahima Diedhiou, Boubacar Demba Ba, Amadou Tahirou Diaw, Alfred Kouly Tine

\section{- To cite this version:}

Tidiane Sané, Catherine Mering, Marie-Christine Cormier-Salem, Ibrahima Diedhiou, Boubacar Demba Ba, et al.. Permanences et mutations dans les terroirs rizicoles de Basse-Casamance (Sénégal). Espace Géographique, 2018, 47 (3), pp.201-218. 10.3917/eg.473.0201 ird-02147673

\section{HAL Id: ird-02147673 https://hal.ird.fr/ird-02147673}

Submitted on 4 Jun 2019

HAL is a multi-disciplinary open access archive for the deposit and dissemination of scientific research documents, whether they are published or not. The documents may come from teaching and research institutions in France or abroad, or from public or private research centers.
L'archive ouverte pluridisciplinaire HAL, est destinée au dépôt et à la diffusion de documents scientifiques de niveau recherche, publiés ou non, émanant des établissements d'enseignement et de recherche français ou étrangers, des laboratoires publics ou privés. 


\section{PERMANENCES ET MUTATIONS DANS LES TERROIRS RIZICOLES DE BASSE-CASAMANCE (SÉNÉGAL)}

SANÉ Tidiane ${ }^{1-2-3}$, Mering Catherine ${ }^{2}$, CORMIER-SALEM Marie-Christine ${ }^{3}$, DiÉDHIOU Ibrahima ${ }^{2}$, BA Boubacar Demba $^{1-3}$, DIAW Amadou Tahirou ${ }^{5}$, TINE Alfred Kouly ${ }^{4}$

tsane@univ-zig.sn

${ }^{1}$ Département de Géographie, UFR Sciences et Technologies, Université Assane Seck de Ziguinchor, BP : 523, Ziguinchor, Sénégal
${ }^{2}$ Université Paris Diderot, Laboratoire Interdisciplinaire des Energies de Demain, UMR CNRS 8236, France
${ }^{3}$ Laboratoire Mixte International Patrimoines et Territoires de l'Eau, UMR 208, PALOC, MNHN, IRD;
${ }^{4}$ Institut National de Pédologie (INP), Institut Sénégalais de Recherche Agricole (ISRA) Sénégal
${ }^{5}$ Laboratoire d'Enseignement et de Recherche en Géomatique, ESP-Université Cheikh Anta Diop de Dakar

Résumé : L'Afrique de l'Ouest subit, depuis plus d'une quarantaine d'années, une dégradation des paysages agricoles. La forte variabilité climatique de ces dernières décennies est un des facteurs déterminants de ces transformations. Elle se traduit par une diminution des précipitations et une importante occurrence des périodes de déficit pluviométrique dont les répercussions sont manifestes sur les systèmes agraires. Les terroirs rizicoles de Basse-Casamance, emblématique des "civilisations agraires" d'Afrique de l'Ouest, traduisent les dynamiques en cours dans les "Rivières du Sud". Ces espaces, qui concentrent de nombreuses activités humaines, présentent de fortes contraintes naturelles et socioéconomiques qui ont des conséquences sur les systèmes rizicoles.

L'analyse des terroirs rizicoles de Basse-Casamance, par une approche des paysages combinant des données géospatiales et des travaux de terrain, montre des dynamiques contrastées entre l'activité rizicole et son environnement biophysique et socio-économique.

L'analyse des photographies aériennes et des images Google Earth, en particulier, fait apparaitre un recul des terroirs rizicoles induit par la forte variabilité climatique, la salinisation et l'acidification des eaux et des terres, et par l'insuffisante main d'œuvre liée à l'exode des jeunes qui se traduit par le manque d'entretien des digues de protection des parcelles rizicoles. Elle révèle ainsi l'existence de fortes interactions entre activités agricoles, écosystèmes exploités et environnement socio-économique.

Mots clés : Afrique de l'Ouest, Basse-Casamance, Dégradation, Déprise rurale, Terroirs rizicoles.

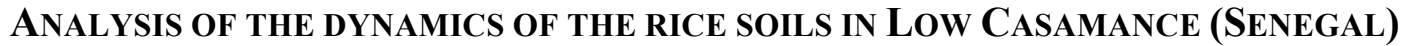

Summary: West Africa undergoes, since more than about forty years, a degradation of the agricultural landscapes. The strong climatic variability of these last decades is one of determining factors of these transformations. It is translated by a decrease of the precipitation and an important case of the periods of rainfall deficit repercussions of which are obvious on the agrarian systems. The rice soils of Low Casamance, a beautiful example of the «agrarian civilizations» of western Africa, translate the current dynamics in the "Rivers of the South". These spaces, which concentrate numerous human activities, present strong natural and socioeconomic constraints, which have consequences on the rice systems.

The analysis of the rice soils of Low Casamance, by an approach of the landscapes combining geospatial data and field works, shows dynamics contrasted between the rice activity and its environment biophysics and socioeconomic.

The analysis, in particular, reveals the backward movement of the rice soils inferred by the strong climatic variability, the salinization and the acidification of waters and lands, and insufficient hand of work (exodus of the young people) resulting in a lack of interview of the dikes of protection of the rice plots of land. It shows that there are interactions between agricultural activities, exploited ecosystems and socioeconomic environment.

Keywords : West Africa, Basse-Casamance, Degradation, Rural Exodus, Rice System, Landscape. 


\section{Introduction}

La Basse-Casamance (fig. 1) appartient à un ensemble géographique dit "Rivières du Sud" (Pélissier, 1966 ; Diop, 1990 ; Cormier-Salem, 1992, 1994 et 1999) qui englobe tous les deltas, les estuaires et les plaines côtières réparties du Saloum au Sénégal à la Sierra-Léone. Cette zone abrite une mosaïque de populations qui y ont construit de véritables polders, défriché la mangrove et dessalé les terres, pour y cultiver du riz (Pélissier, 1966; Marius, 1985 ; Cormier-Salem, 1995, 1999). Les Diola de Basse Casamance font partie de ces populations côtières-atlantiques d'Afrique de l'Ouest qui ont façonné les paysages littoraux et $\mathrm{y}$ ont imprimé une véritable tradition rizicole qualifiée par Pélissier (1966)"d'authentique civilisation agraire". Les systèmes de production rizicole se traduisent par leur caractère traditionnel car les processus techniques reposent encore essentiellement sur l'énergie humaine et la prédominance de l'utilisation d'un outillage manuel, le kajend $u^{l}$.

L'appartenance de la Basse-Casamance au domaine climatique sud-soudanien lui confère des caractéristiques climatiques et morpho-pédologiques particulières. Première région pluviométrique du Sénégal (plus $1000 \mathrm{~mm}$ par an), elle possède un réseau hydrographique relativement dense et l'une des couvertures végétales les plus importantes du pays. Elle a longtemps été perçue comme le "grenier agricole" qui pouvait potentiellement subvenir à la forte demande en riz du pays. La riziculture inondée, historiquement implantée dans la zone estuarienne du fleuve Casamance, portait en elle de grands espoirs en termes d'autosuffisance alimentaire (Montoroi, 1996). Ainsi, la Basse-Casamance a longtemps joui d'une réputation de prospérité. Mais elle se trouve désormais dans une situation de profondes mutations écologiques et socio-économiques. La sécheresse des années 1970 et 1980, et l'instabilité actuelle du régime pluviométrique ont provoqué une transformation profonde de l'environnement naturel et des formes de gestion qui s'y appliquent que nous efforcerons de mettre en évidence par l'analyse des données climatiques, pédologiques et l'interprétation de photographies aériennes et Google Earth autour de terroirs agricoles emblématiques des changements qu'a connu la région depuis la fin des années 1960. Les mutations environnementales et socioéconomiques conduisent les populations à l'exode rural contribuant ainsi à la fragilisation des systèmes de production agricole (Cormier-Salem, 1985, 1992 ; Barbier-Wiesser, 1994 ; Chéneau-Loquay, 1994a et b ; Grdr et al., 2017 ; Cormier-Salem et Sané, 2017 ; Sané et al., 2017). Malgré de telles évolutions qui se conjuguent avec une situation d'insécurité aux frontières liée au conflit armé qui a débuté dans les années 1980, les populations locales parviennent à développer des stratégies d'adaptation en diversifiant leurs activités et en revalorisant les anciens usages de la mangrove (pêche artisanale itinérante, récolte d'huîtres, etc.) et en développant d'autres sources de revenus comme les plantations d'anacardier, le maraîchage, et les dynamiques migratoires, de plus en plus lointaines et longues.

\footnotetext{
${ }^{1}$ Instrument aratoire utilisé par les Diola de Basse-Casamance. Il s'agit d'une longue pelle oblongue bien adaptée aux terres argileuses.
} 


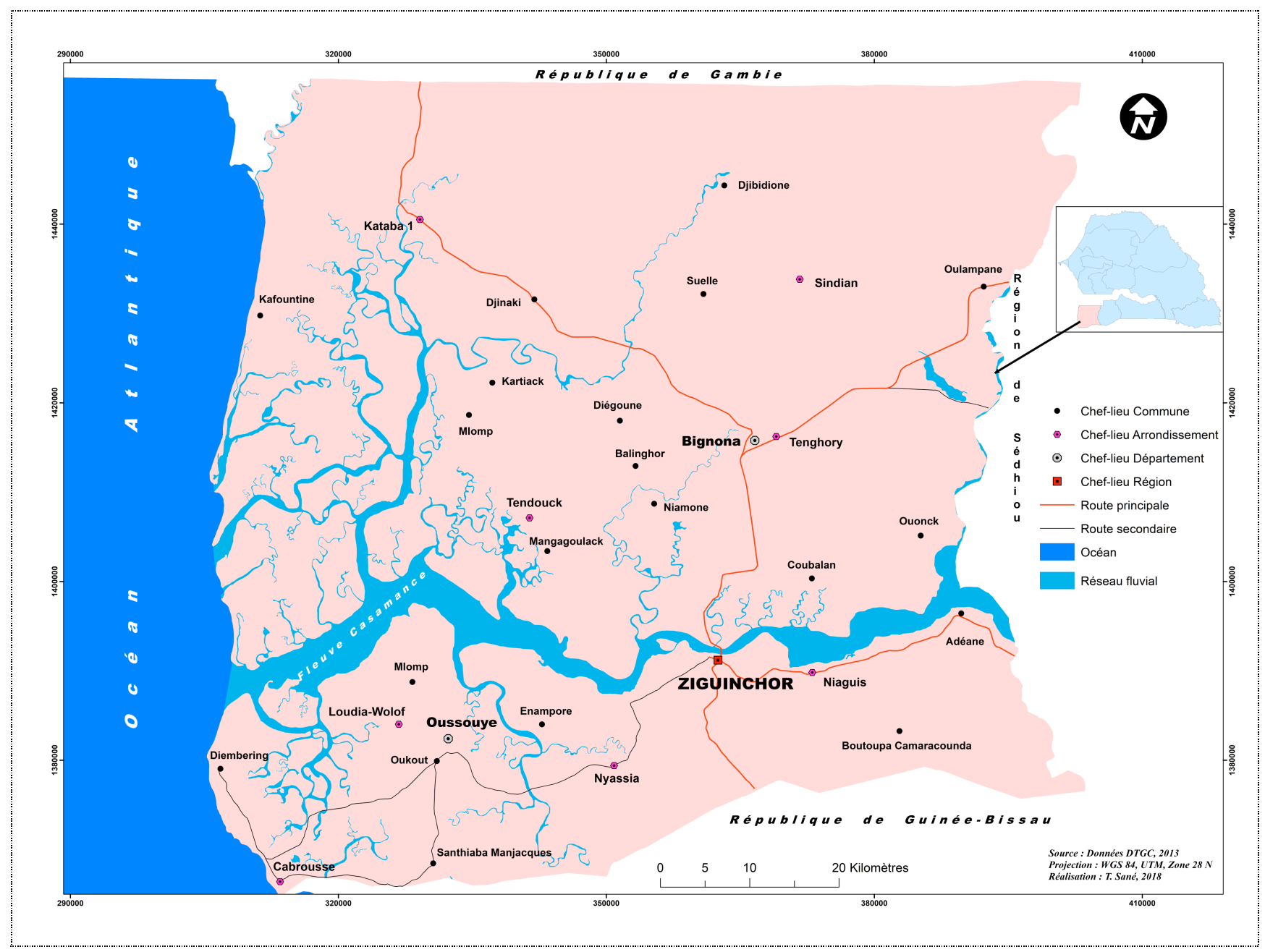

Figure 1 : La Basse-Casamance dans l'espace sénégalais

\section{I - Données et méthodes}

Cette étude a nécessité l'usage des données pluviométriques (pluies journalières, mensuelles et annuelles) et le prélèvement d'échantillons d'eaux et de sols pour caractériser l'environnement biophysique de la Basse-Casamance. L'analyse de la dynamique des terroirs rizicoles des sites retenus a été réalisée au moyen de photographies aériennes de 1969 (mission AOF, ND28-I-III/500) et d'images Google Earth de 2015.

La démarche méthodologique comporte trois volets. Pour l'analyse des données pluviométriques, des calculs statistiques simples ont été utilisés. La détermination de la durée de la saison des pluies a été réalisée selon les méthodes Gueye et Sivakumar (1992) et Sané et al. (2008).

Les échantillons de sol et d'eau ont fait l'objet de prélèvement et d'analyse physico-chimiques au Laboratoire National de Pédologie (INP), (fertilité physique et chimique : texture, degré de salinité et teneur en éléments physiques et chimiques). Le prélèvement a été réalisé sur un certain nombre de sites où nous avons identifié la zone de rizière, celle de la forêt, où le couvert végétal est intact, et la zone de culture où sont menées des activités agricoles pluviales. Le repérage in situ de la toposéquence a permis de positionner trois points de prélèvement : P1 dans la vallée (zone de marnage ou tannes), $\mathbf{P 2}$ (zone de rizière) et $\mathbf{P 3}$ (zone de plateau) conformément à la toposéquence et selon les modes d'occupation des sols. Sur chacun des points de sondage, nous avons prélevé deux échantillons de sols, à des profondeurs de 0-20 $\mathrm{cm}$ et 20-40 cm. Enfin la cartographie de l'occupation des sols des terroirs 
rizicoles a été réalisée par photo-interprétation. Nous avons également mis à contribution et mobilisé divers outils, des enquêtes auprès des paysans (Sané, 2017) à la cartographie participative (Sané et al., 2017 ; Cormier-Salem et Sané, 2017 ; Ehemba et al., 2017 ; Grdr et al., 2017), des suivis, des observations sur le terrain.

\section{II - La place de la riziculture et du riz dans la société diola}

A l'échelle mondiale, la gestion des espaces littoraux des "Rivières du Sud" présente une forte originalité, liée en particulier à l'importance et à l'ancienneté de la riziculture de mangrove (CormierSalem, 1995). La Basse-Casamance, peuplée en majorité par les Diola (61\% de la population de la région), est connue pour être, avec le Delta Intérieur du Niger, l'un des plus anciens foyers de la riziculture en Afrique observés depuis 1500 avant J.-C. (Cormier-Salem, 1992 et 1999 ; Bezançon, 1994). Peuples riziculteurs majoritaires en Basse-Casamance, les Diola constituent un rameau important de la vieille civilisation agraire qui a longtemps vécu sur les littoraux ouest-africains et qui est très profondément attachée à ce milieu naturel prospère auquel ils se sont adaptés depuis de nombreux siècles. Mark (1985) et Roche (1985) situent leur installation dans la région avant le XVI ${ }^{\text {ème }}$ siècle.

Les paysans diola sont réputés pour leurs techniques très élaborées d'aménagement en rizières de mangrove. Cette vocation rizicole est intimement liée à leur histoire (Portères, 1952; Thomas, 1959 ; Linares, 1970 ; Gourou, 1984 ; Montoroi, 1996 ; Diédhiou, 2001). Ils ont ainsi acquis, au cours du temps, un remarquable savoir agricole et piscicole, en mettant en place un dispositif de digues et de diguettes pour le contrôle des eaux et servant également de limites des propriétés foncières (photo 1). Ce dispositif a été ingénieusement mis en place et perpétué à travers des générations. Il est à l'origine de l'émergence d'une riziculture de subsistance remarquable transmise et diffusée dans tout le littoral ouest-africain, de la Guinée-Bissau à la Sierra-Leone (Montoroi, 1996; Grdr et al., 2017) où on retrouve une population (cas des Baga en Guinée et des Temne en Sierra-Léone) aux pratiques rizicoles plus ou moins similaires à celles des Diola de Basse Casamance.

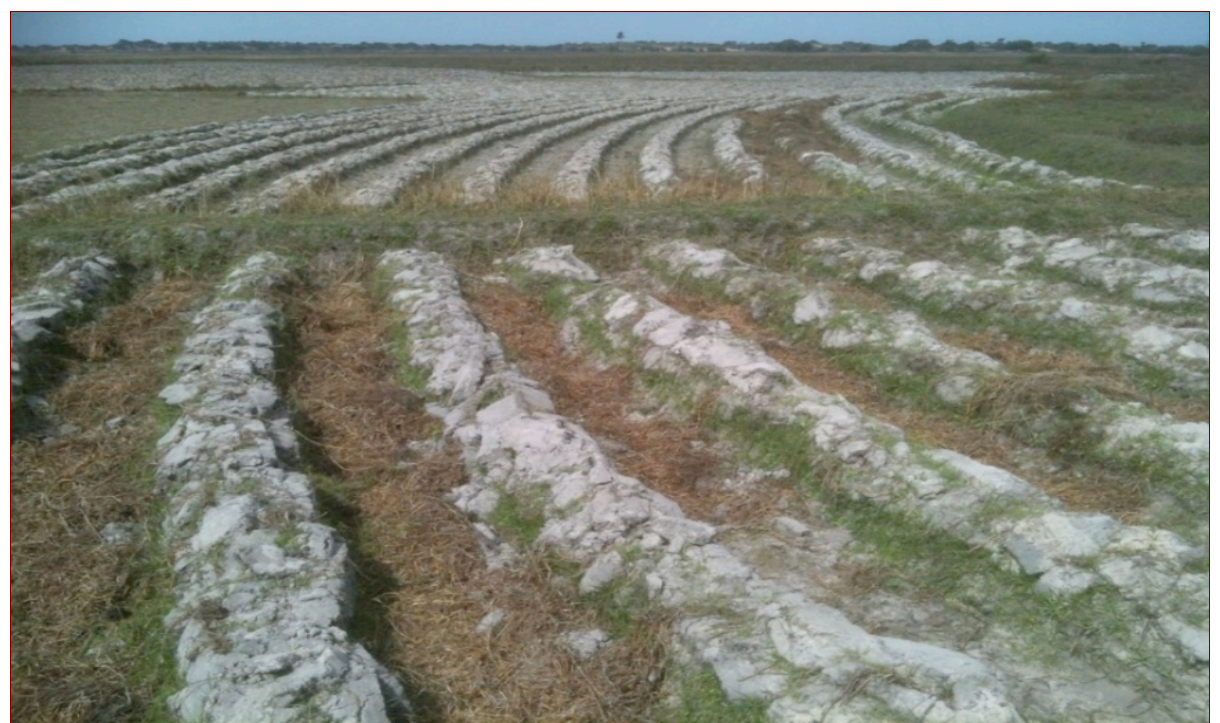

Photo1 : Dispositif de digues et diguettes dans une vallée rizicole de Basse-Casamance

En pays diola, la production de riz demeure l'objectif prioritaire. En effet, le riz permet non seulement de couvrir les besoins alimentaires mais il est également source de richesse et de prestige dont témoignaient jadis les greniers remplis de riz consommé ou utilisé lors des cérémonies traditionnelles et religieuses.

Pélissier (1966) et Gourou (1984) affirment à ce sujet que pour les Diola, manger n'a d'autre sens que manger du riz. Par ailleurs, le calendrier des activités rizicoles rythme toute la vie de la communauté paysanne tandis que l'organisation du terroir reste articulée autour des bas-fonds rizicultivables. Tous 
les auteurs s'accordent ainsi à reconnaître la place majeure du riz dans le paysage, l'alimentation, l'organisation du terroir villageois, le calendrier des activités, les rites et les mythes, faisant ainsi de la Basse-Casamance une région à vocation rizicole avec cependant d'importantes mutations environnementales et socioéconomiques, dans le contexte actuel (Cormier-Salem, 1999 ; Sané, 2017 ; Sané et al., 2017 ; Ehemba et al., 2017 ; Cormier-Salem et Sané, 2017 ; Grdr et al., 2017).

\section{III - Les systèmes d'exploitation rizicoles de Basse Casamance : un environnement en transition}

L'analyse des grandes tendances de la dynamique des systèmes de production rizicole en BasseCasamance révèle leurs recompositions actuelles, dont le processus est lié à un ensemble de facteurs à la fois naturels et anthropiques.

\section{Un environnement physico-géographique contraignant}

Les zones rizicoles de Basse-Casamance résultent pour l'essentiel du défrichement de la mangrove à laquelle se sont substitués des casiers rizicoles depuis des centaines d'années. Cette forme de mise en valeur s'observe sur l'ensemble de l'estuaire du fleuve Casamance, depuis l'embouchure jusqu'aux moindres ramifications du réseau hydrographique. Au contact avec les plateaux, les conditions d'exploitation changent sensiblement car, outre les ressources qu'offre la mangrove proche, les paysans diola exploitent d'autres ressources forestières, ce qui leur permet de pallier les mauvaises années de récolte occasionnées par les aléas climatiques. Des considérations socio-historiques soulignent l'originalité des implantations humaines en Basse-Casamance à travers la dualité des terroirs agricoles caractérisées par l'exploitation à la fois des bas-fonds et des plateaux. Les activités agricoles bénéficient ainsi de l'influence quotidienne des marées et de l'inondation annuelle par les pluies (Pélissier, 1966 ; Marzouk, 1991 et 1980 ; Cormier-Salem, 1992, 1994, 1995, 1999 ; Cormier-Salem et Sané, 2017 ; Sané, 2017). Cependant, à l'épreuve des changements globaux actuels, les zones rizicoles de Basse Casamance sont soumises à des mutations profondes.

\section{1 -Une pluviométrie très instable}

La forte variabilité du régime pluviométrique observée depuis les années 70 rend aujourd'hui plus difficile la pratique de la riziculture en Basse-Casamance alors que jusqu'à la fin des années soixante les conditions environnementales étaient tout à fait adaptées à cette culture. La disponibilité en eau, la nature et les aptitudes des sols sont actuellement influencées, d'une part par une pluviométrie relativement moins importante et surtout plus irrégulière que par le passé et, d'autre part, par le contraste entre les bas plateaux cloisonnés et les bas-fonds influencés par l'intrusion de l'eau de mer. L'instabilité pluviométrique s'exprime à travers la distribution mensuelle des pluies, la durée de la saison des pluies (fig. 2) et leur évolution interannuelle (fig. 3). Cette instabilité est matérialisée par une succession d'années sèches et d'années humides et une répartition mensuelle inégale des volumes pluviométriques qui influence fortement la durée de la saison pluvieuse dont le raccourcissement est manifeste depuis les années de sécheresse. L'irrégularité spatio-temporelle des pluies observée en Basse-Casamance ne fait qu'accentuer la dépendance de la vie rurale à leur égard, en induisant notamment des risques d'une réduction progressive de la gamme des plantes cultivables (Sané, 2017). 


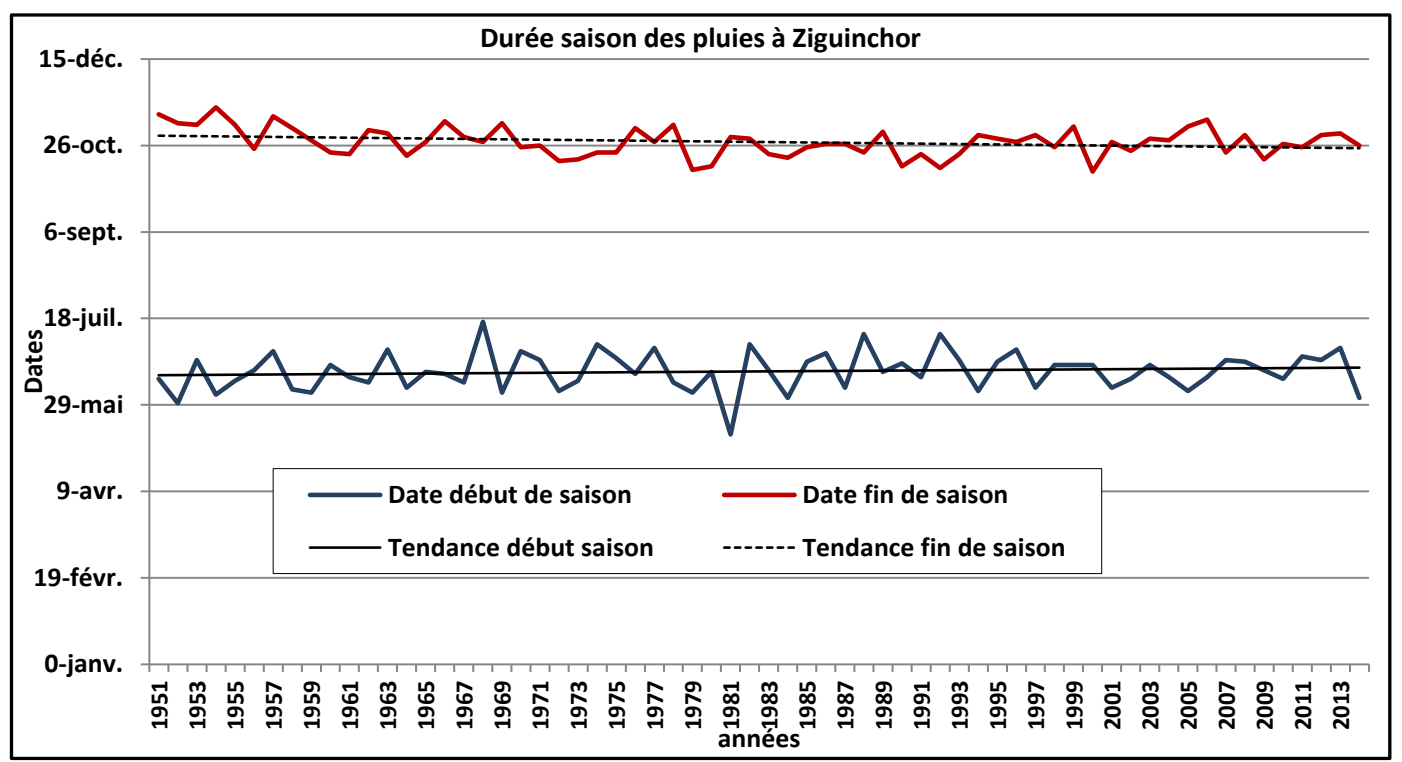

Figure 2 : Durée de la saison des pluies à Ziguinchor (Sané, 2017)

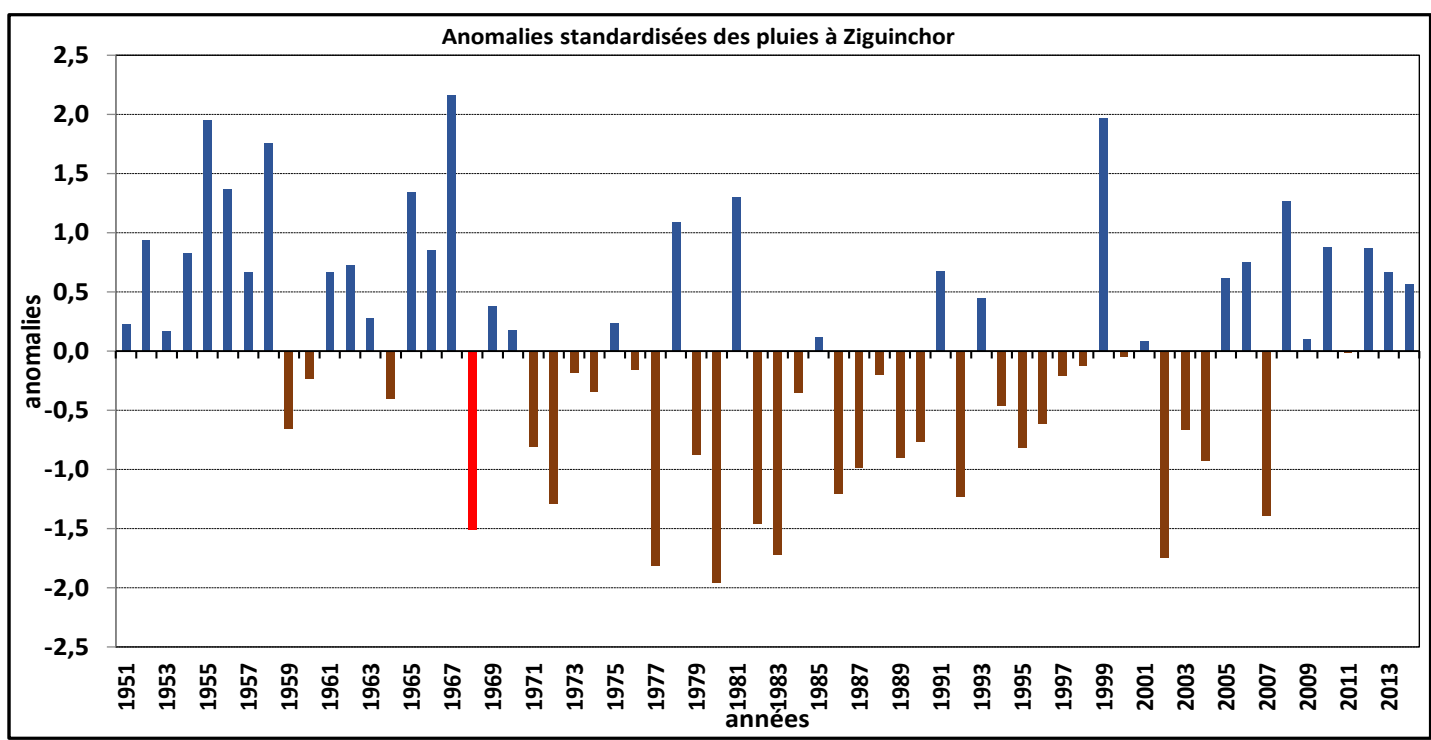

Figure 3 : Evolution interannuelle des pluies à Ziguinchor : rupture intervenue en 1968 (Sané, 2017)

Malgré l'instabilité climatique actuelle, la Basse-Casamance demeure la région la plus arrosée du Sénégal, les précipitations étant supérieures à $1000 \mathrm{~mm}$ pour l'essentiel des stations pluviométriques. Cependant, cette forte variabilité pluviométrique a des répercussions sur l'environnement biophysique, notamment sur les eaux et les sols.

\section{2 -Des caractéristiques pédologiques et hydrologiques dégradées}

La péjoration climatique, survenue depuis la fin des années 60, s'est manifestée en Basse-Casamance par une salinisation et une acidification des rizières de mangrove devenues de ce fait de moins en moins productives. En effet, les terres rizicoles des bas-fonds ont été progressivement contaminées par les eaux de surface sursalées (Marius, 1985 ; Boivin, 1991; Mougenot et al., 1990). L'analyse des paramètres pédologiques s'est faite ici sur la base d'un certain nombre de sites répartis selon les systèmes agraires (fig. 4). Les sites de Thionck-Essyl au nord, Coubanao à l'est et Diembering au sudouest sont retenus. 


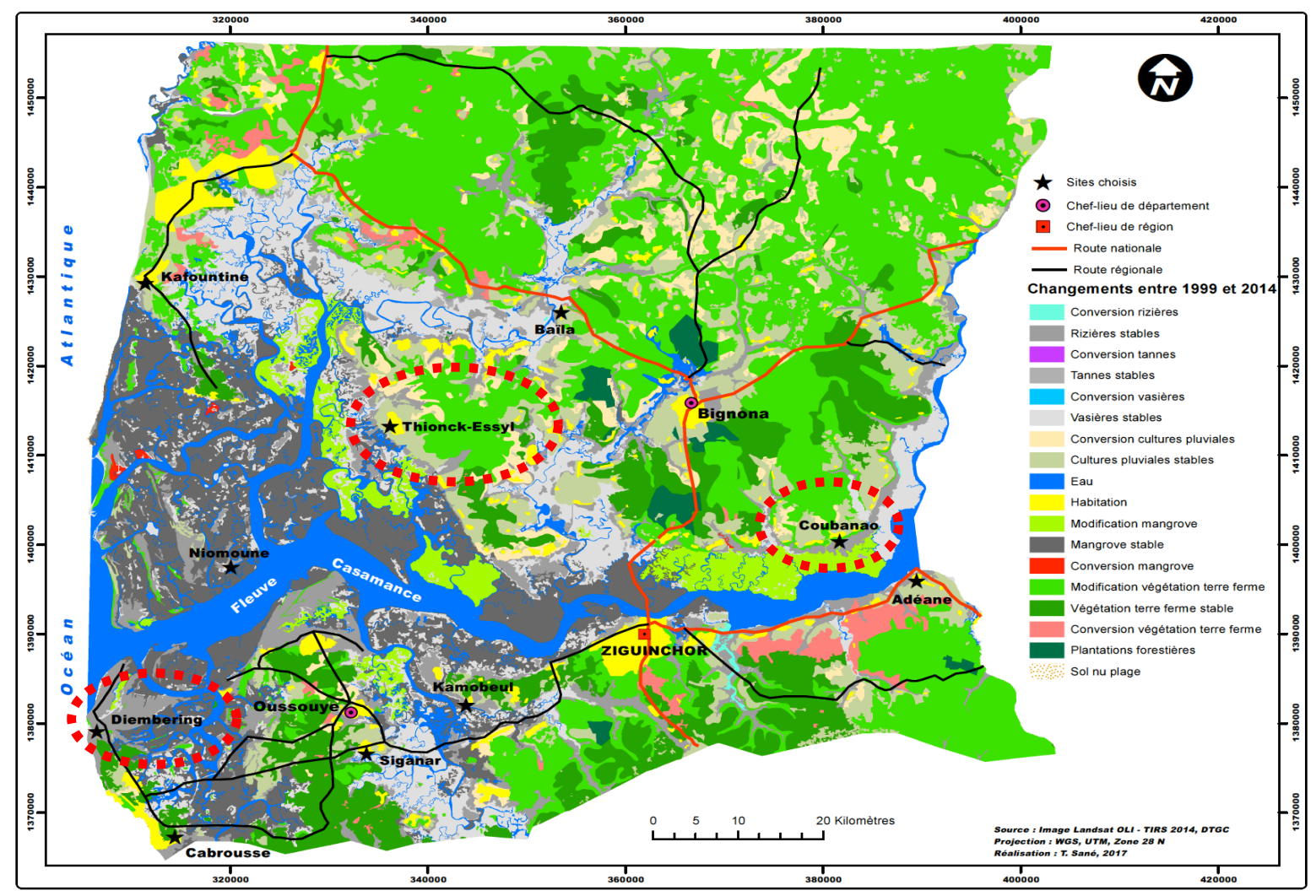

Figure 4 : Localisation des terroirs rizicoles étudiés

A Thionck-Essyl, les sols de vasières anciennes hydromorphes sont observés dans les rizières. Ces sols sont non salins et très sableux avec un taux d'argile compris entre 2 et $9 \%$, exceptionnellement de $13 \%$. Ils sont moyennement riches en matières organiques et leur $\mathrm{pH}$ est légèrement acide (fig. 5).Des cultures de riz y sont portées pendant la saison des pluies mais, pour leur meilleure productivité, des aménagements permettant d'assurer le contrôle de l'apport en eau douce sont indispensables.

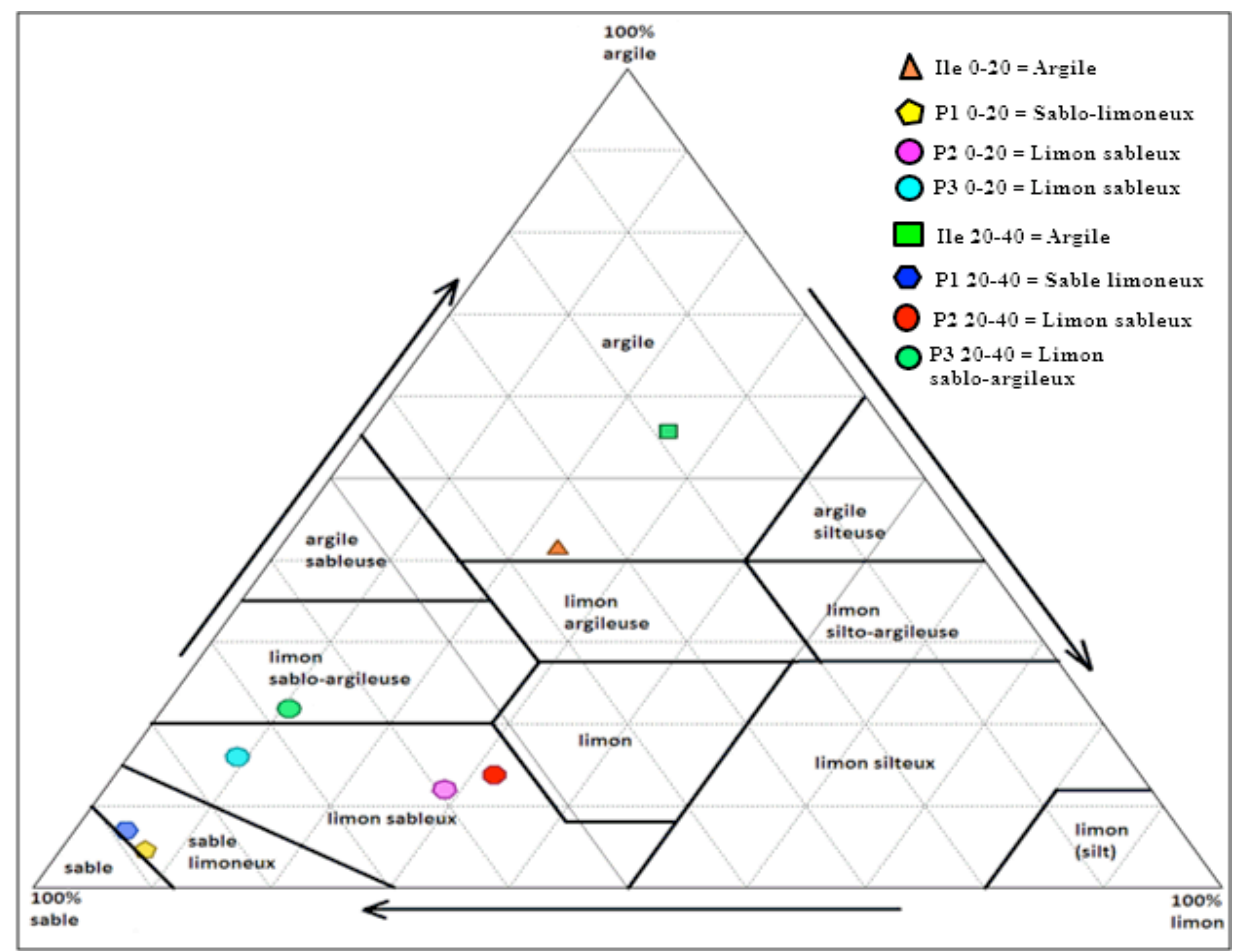

Figure 5 : Diagramme textural des sols de de Thionck-Essyl 
A Coubanao, on note la présence de sols de terrasses colluvio-alluviales sur matériau colluvial peu évolués à texture sableuse et limono-sableuse. Les textures argileuse, limono-sableuse et sablolimoneuse indiquent des phénomènes d'ensablement remarquables au niveau des bas-fonds (fig. 6). Le $\mathrm{pH}$ est très acide (inférieur à 4) mais relativement riche en matières organiques dont le taux dépasse $10 \%$ à 1 'horizon $0-20 \mathrm{~cm}$. La capacité d'échanges cationiques est très élevée mais le taux de saturation en bases est très faible. Ces sols sont aptes à porter plusieurs cultures mais il faudra, apporter des amendements pour améliorer leur fertilité. La forte acidité de ces sols constitue en effet une contrainte pour le développement rizicole.

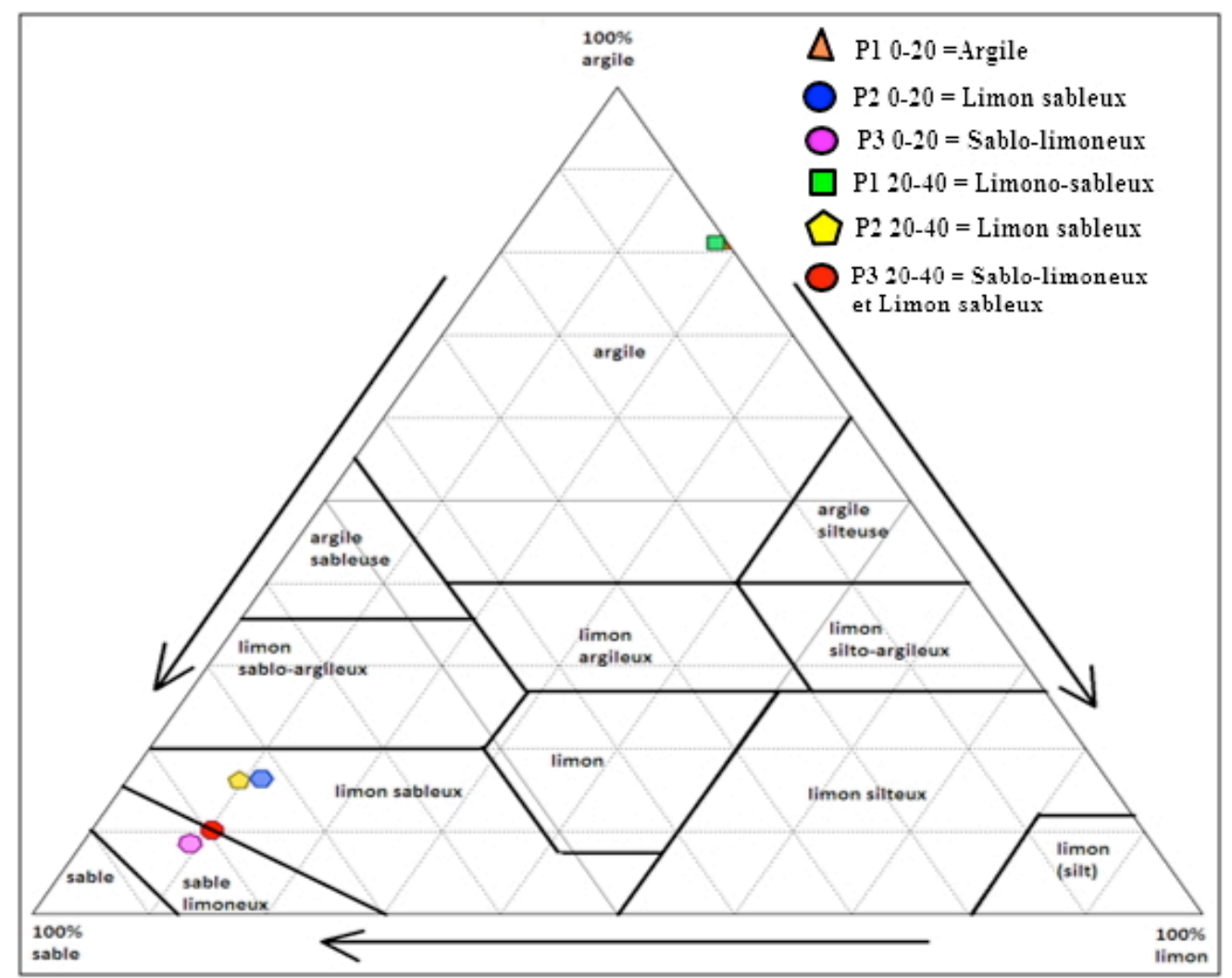

Figure 6 : Diagramme textural des sols à Coubanao

A Diembering située en zone littorale, on rencontre des sols peu évolués d'apport des cordons littoraux récents (fig. 7). Ces sols sont sableux à plus de 70\%, non salés mais légèrement acides, riches en matières organiques avec une capacité d'échange cationique très élevée. Ce sont des sols qui n'offrent pas de grandes possibilités agricoles, notamment rizicoles, à cause de leur texture trop sableuse. Néanmoins, ces sols restent aptes au développement du maraîchage. Cette activité est développée dans la plupart des terroirs villageois, à l'image de la zone littorale (Cabrousse, Cap-Skirring, Diembering, Kafountine) où les infrastructures touristiques participent au développement de la filière horticole. 


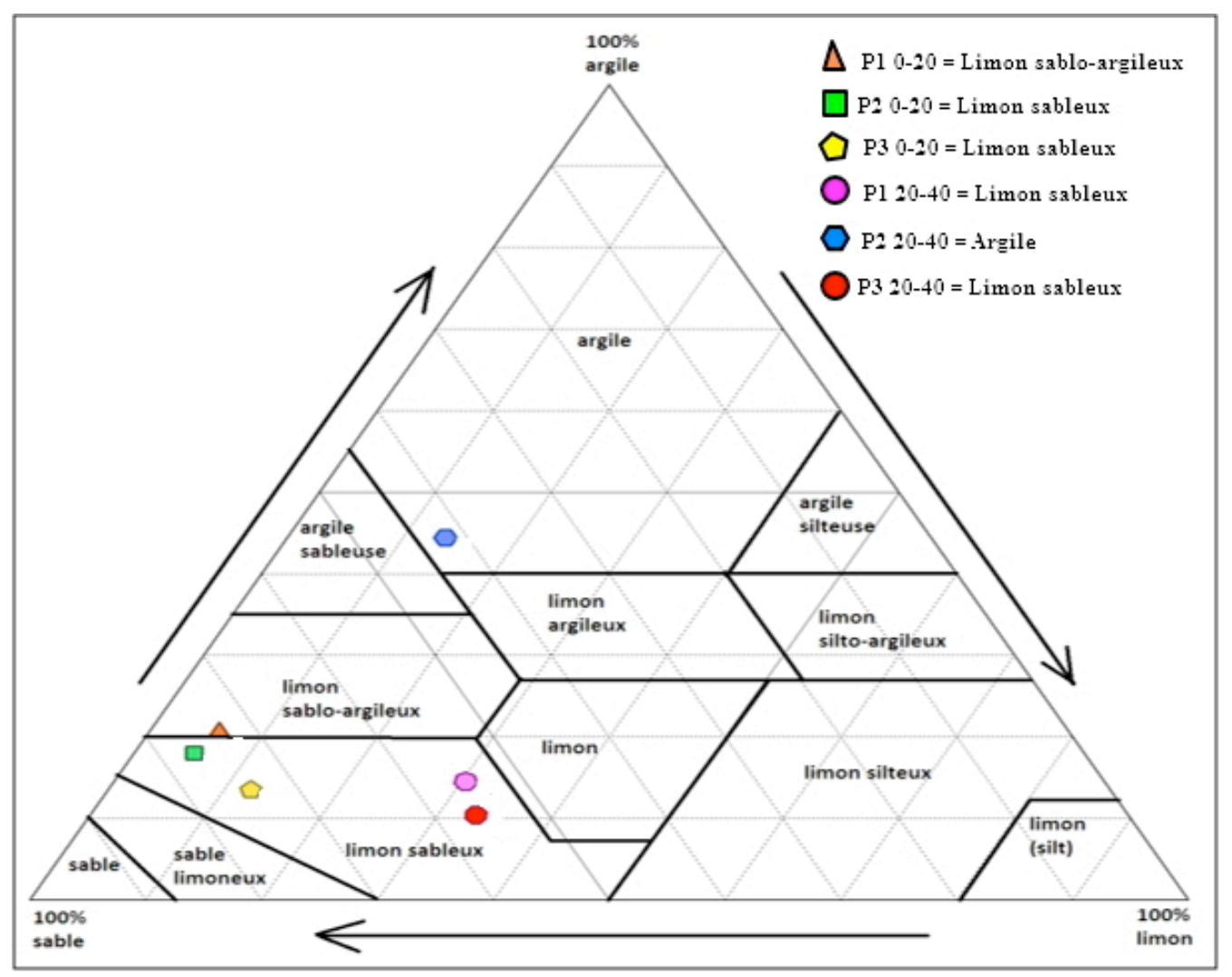

Figure 7 : Diagramme textural des sols à Diembering

Les caractéristiques édaphiques des sols de rizières dont les textures sont favorables à la rétention d'eau, conditionnant ainsi le bon développement du riz, sont, dans le cas où nous observons des phénomènes d'ensablement dans tous les sites, dénaturées par la présence de niveaux sableux subaffleurants. La salinité et l'ensablement constituent les types de dégradation des sols les plus communs. Leurs effets ont un impact sur la fertilité des sols et sur leur aptitude à la riziculture.

Les résultats des mesures des ions chlorures $\left(\mathrm{Cl}^{-}\right)$confirment le caractère excessivement salé des fleuves et celui moyennement salé des eaux de puits. Ils montrent que la dureté (somme des concentrations de Calcium et Magnésium) est excessive pour toutes les eaux de marigots alors qu'elle est faible pour les puits. Les teneurs en sodium $\left(\mathrm{Na}^{+}\right)$, sulfates $\left(\mathrm{SO}_{4}{ }^{2-}\right)$ et potassium $\left(\mathrm{K}^{+}\right)$confirment la forte minéralisation des eaux de marigots et la minéralisation moyenne des eaux de puits (fig. 8). 


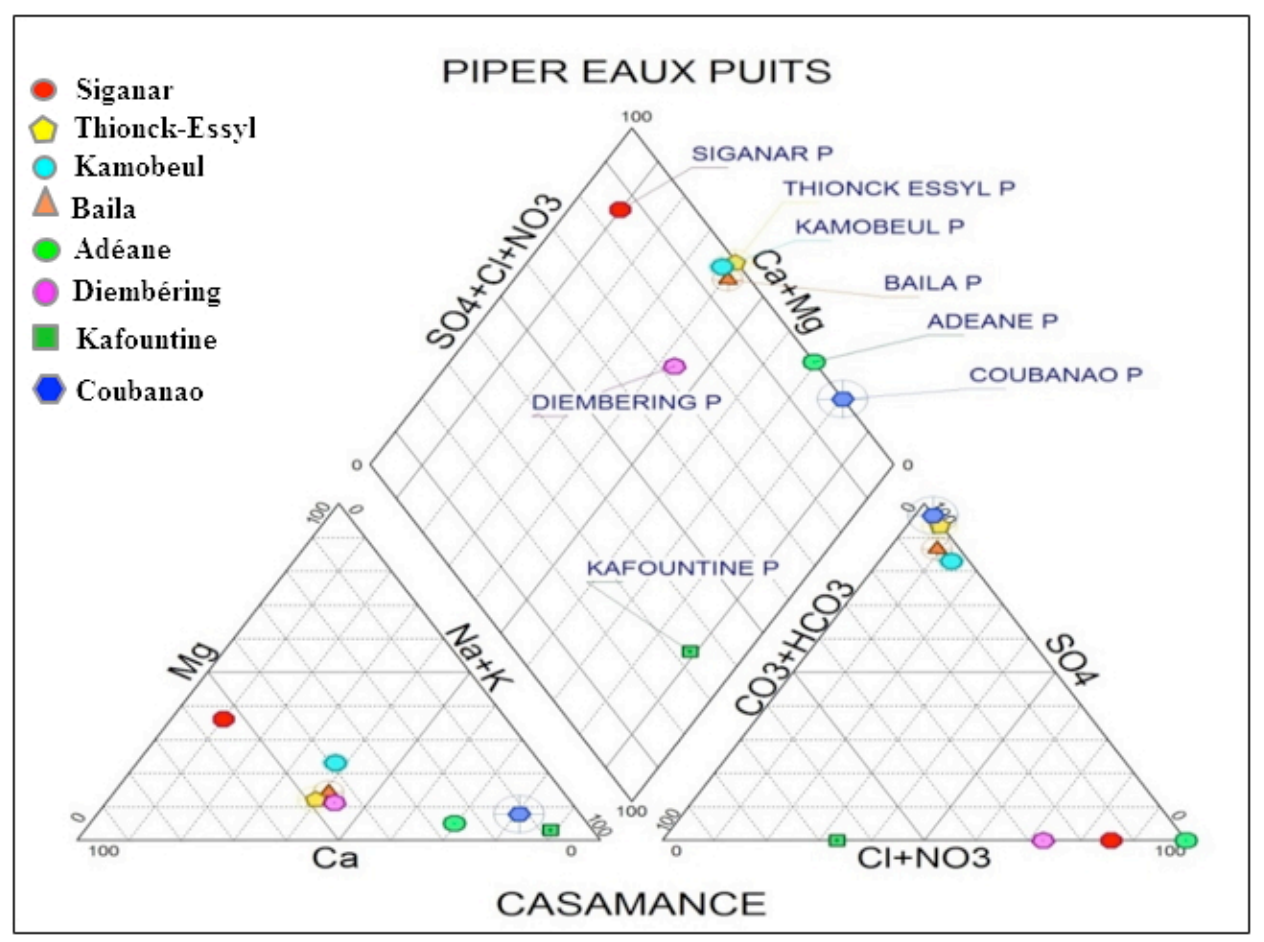

Figure 8 : Caractéristiques physico-chimiques des eaux des nappes des sites étudiés

Les problèmes de l'environnement biophysique ne sont pas les seules contraintes pour le développement rizicole. La riziculture de Basse-Casamance fait également face au problème de la faiblesse de main d'œuvre.

\section{Une main d'auvre de moins en moins disponible}

Etant saisonnier au départ, l'exode rural est un phénomène ancien en Basse-Casamance (Pélissier, 1966 ; Loquay, 1979 ; Harza, 1981 ; Cormier-Salem, 1985 ; Bonnefond et Loquay, 1985) mais qui a pris de l'ampleur ces dernières décennies (Grdr et al., 2017; Diop, 2017) comme le suggère le tableau 1. Son développement est lié à l'urbanisation généralisée, à l'amélioration des communications (ouverture de la route Dakar-Ziguinchor en 1952), à l'importance de la scolarisation en Basse-Casamance (taux brut dépassant $100 \%$ ), à l'intégration à l'économie de marché et à la forte instabilité climatique. Les jeunes qui partent en ville pour diverses raisons reviennent moins souvent qu'auparavant. En effet, les agglomérations urbaines leur offrent plus de possibilités d'emplois moins contraignants que les activités rizicoles caractérisées par la pénibilité des travaux rizicoles (Bonnefond et Loquay, 1985 ; Cormier-Salem, 1985 et 1992 ; Sané, 2017 ; Cormier-Salem et Sané, 2017 ; Ehemba et al., 2017).

Tableau $1:$ Nombre de personnes émigrées par ménage dans le Bandial

\begin{tabular}{|l|r|r|}
\hline Nombre de personnes émigrées par ménage & Nombre de citations & \multicolumn{2}{l|}{$\%$} \\
\hline Sans réponse & 7 & 2,8 \\
\hline Moins de 2 & 37 & 14,9 \\
\hline De 2 à 4 & 77 & 31,0 \\
\hline De 4 à 6 & 67 & 27,0 \\
\hline De 6 à 8 & 30 & 12,1 \\
\hline De 8 à 10 & 17 & 6,9 \\
\hline De 10 à 12 & 5 & 2,0 \\
\hline 12 et plus & 8 & 3,2 \\
\hline Total & 248 & 100 \\
\hline
\end{tabular}


Depuis de nombreuses décennies, il était d'usage que les jeunes partent pour la ville, notamment à Dakar pendant la saison sèche et qu'ils reviennent en saison des pluies pour aider aux travaux agricoles. Or, depuis les années 70 et 80 , dans un contexte où la sévérité des déficits pluviométriques avait profondément bouleversé les campagnes sénégalaises par l'absence ou la faiblesse de récoltes, le tarissement précoce des mares, les difficultés d'approvisionnement en eau, la perte du cheptel, la disette dans les foyers, le phénomène de migration vers des lieux aux conditions plus favorables, particulièrement vers les villes s'est amplifié (Fall et al., 2005). Au cours de cette même période la scolarisation s'est accrue en Casamance avec la multiplication des collèges et des lycées, la création de l'université Assance Seck et d'autres structures d'enseignement supérieur... Dans un tel contexte, les jeunes ont progressivement rompu avec l'habitude de revenir dans leur village durant l'hivernage ; ce qui a eu pour conséquence d'accroître la surcharge de travail pour ceux qui y sont restés. Si la migration apparaît comme une stratégie partielle d'adaptation permettant de profiter d'autres opportunités, elle constitue, au moment même où l'amélioration des conditions climatiques devrait leur être favorable, un sérieux handicap pour les activités rizicoles qui nécessitent des travaux d'entretien des digues et diguettes en saison sèche pour éviter l'envahissement des parcelles par l'eau de mer. Ainsi, les causes et les conséquences de la forte variabilité pluviométrique et de l'exode rural conditionnent aujourd'hui fortement les pratiques agricoles et sociales en Basse-Casamance où la force de travail humaine était jusqu'à présent la source d'énergie quasi-unique pour assurer la mise en valeur des parcelles rizicoles (Pélissier, 1966 ; Cormier-Salem, 1985 et 1992 ; Grdr et al., 2017 ; Sané, 2017).

La composition et l'évolution de la population active ${ }^{2}$ disponible sont des facteurs à prendre en compte pour évaluer les chances d'une reprise de la riziculture en Basse Casamance. En effet, la force de travail humaine qu'exigent les travaux rizicoles dépend, encore et dans une large mesure, de la mise en culture et de l'entretien des rizières. La population active ou main d'œuvre est globalement constituée de personnes âgées de 15 à 64 ans. Au regard des chiffres de la population comprise dans cette fourchette d'âge (tabl. 2), la Basse Casamance dispose d'une population active de 48,5\% par rapport à la population totale. Il faut cependant soustraire de ce pourcentage non seulement la population active urbaine mais aussi les étudiants et les élèves habitant officiellement en zone rurale.

Tableau 2 : Répartition de la population selon l'âge et le sexe

\begin{tabular}{|l|r|r|r|}
\hline Groupes d'âge & Hommes & Femmes & Total \\
\hline $0-4$ & 40670 & 39822 & 80492 \\
\hline $5-9$ & 36341 & 33430 & 69771 \\
\hline $10-14$ & 31531 & 26181 & 57712 \\
\hline $15-19$ & 24068 & 20988 & 45056 \\
\hline $20-24$ & 16933 & 17688 & 34621 \\
\hline $25-29$ & 12959 & 14039 & 26998 \\
\hline $30-34$ & 10179 & $1111 \mathrm{f}$ & 21292 \\
\hline $35-39$ & 8746 & 10629 & 19375 \\
\hline $40-44$ & 6888 & 8025 & 14913 \\
\hline $45-49$ & 7513 & 8676 & 16189 \\
\hline $50-54$ & 6102 & 6159 & 12261 \\
\hline $55-59$ & 6480 & 7453 & 13933 \\
\hline $60-64$ & 4629 & 4484 & 9113 \\
\hline $65-69$ & 4435 & 4354 & 8789 \\
\hline 70 et + & 5352 & 5124 & 10476 \\
\hline Total & 222826 & 218165 & 440991 \\
\hline
\end{tabular}

\footnotetext{
${ }^{2}$ La population active peut être définie ici comme toute personne, de sexe masculin ou féminin d'un âge donné, susceptible de fournir, durant une période déterminée, une main-d'œuvre nécessaire aux activités de production rizicole.
} 
Le taux et le niveau de scolarisation étant de plus en plus élevés, les jeunes scolarisés ne reviennent pas s'installer dans le village pour devenir agriculteur. Ils veulent désormais se construire une trajectoire différente de celle de leurs parents et deviennent plus exigeants quant à leurs conditions de vie. Leur départ vers les milieux urbains a des conséquences directes et indirectes sur la production agricole. C'est pourquoi, dans la classe des actifs permanents ruraux, on ne retrouve souvent que les jeunes mariés et agriculteurs qui ont fait le choix de rester au village, probablement pour des raisons sociales. C'est dans ce contexte, avec la remontée de la langue salée, que certaines parcelles rizicoles sont devenues incultivables, ce qui amplifie la dégradation des paysages rizicoles. Il faut cependant noter que les effets néfastes de l'exode rural des jeunes sur l'activité agricole peut parfois être compensé au niveau de l'exploitation familiale par l'envoi d'argent aux parents restés au village pour leur permettre de payer des "bras disponibles" (ouvriers agricoles en provenance de la Guinée-Bissau, associations de jeunes des villages, etc.) mais aussi d'assurer certaines dépenses relatives aux charges sociales dans un milieu en recomposition.

\section{Des terroirs rizicoles en mutation}

La dynamique de l'environnement biophysique en Basse-Casamance a provoqué un recul des parcelles rizicoles. L'analyse diachronique de l'occupation des sols des terroirs rizicoles entre 1969 et 2015 montre, sur l'ensemble des sites étudiés, le recul des superficies rizicultivables. Ainsi, à ThionckEssyl, les superficies ont régressé d'environ 11,4\%. La mangrove a perdu 22,4\% de sa superficie (tabl. 3). La perte des terres rizicoles se manifeste également à travers les rizières non fonctionnelles c'est-àdire des parcelles devenues incultes (fig. 9). Le développement des superficies de tannes $(+105,9 \%)$ constitue également un indicateur de dégradation des terroirs rizicoles et de la mangrove.

Tableau 3 : Evolution des statistiques d'occupation des sols à Thionck-Essyl entre 1969 et 2015

\begin{tabular}{|l|c|c|c|c|c|}
\hline Unités paysagères & $\begin{array}{c}\text { Superficies } \\
\text { en 1969 } \\
\text { (ha) }\end{array}$ & $\begin{array}{c}\text { Superficies } \\
\text { en \% }\end{array}$ & $\begin{array}{c}\text { Superficies } \\
\text { en 2015 } \\
\text { (ha) }\end{array}$ & $\begin{array}{c}\text { Superficies } \\
\text { en \%o }\end{array}$ & $\begin{array}{c}\text { Progression/régression } \\
\text { (en \%) }\end{array}$ \\
\hline Cours d'eau & 1443,5 & 14,4 & 1442,6 & 14,4 & $-0,1$ \\
\hline Habitation & 210 & 2,1 & 633,2 & 6,3 & 201,5 \\
\hline Mangrove & 4584,6 & 45,6 & 3557,1 & 35,4 & $-22,4$ \\
\hline Rizières & 1387,5 & 13,8 & 1229,9 & 12,2 & $-11,4$ \\
\hline $\begin{array}{l}\text { Rizières non } \\
\text { fonctionnelles }\end{array}$ & - & - & 4,5 & 0,04 & - \\
\hline Tannes & 1131,7 & 11,3 & 2330,5 & 23,2 & 105,9 \\
\hline $\begin{array}{l}\text { Végétation de terre } \\
\text { ferme }\end{array}$ & 64,9 & 0,6 & 424,8 & 4,2 & 554,5 \\
\hline Cultures de plateau & 1230 & 12,2 & 429,8 & 4,3 & $-65,1$ \\
\hline Total & $\mathbf{1 0 0 5 2 , 3}$ & $\mathbf{1 0 0}$ & $\mathbf{1 0 0 5 2 , 3}$ & $\mathbf{1 0 0}$ & - \\
\hline
\end{tabular}



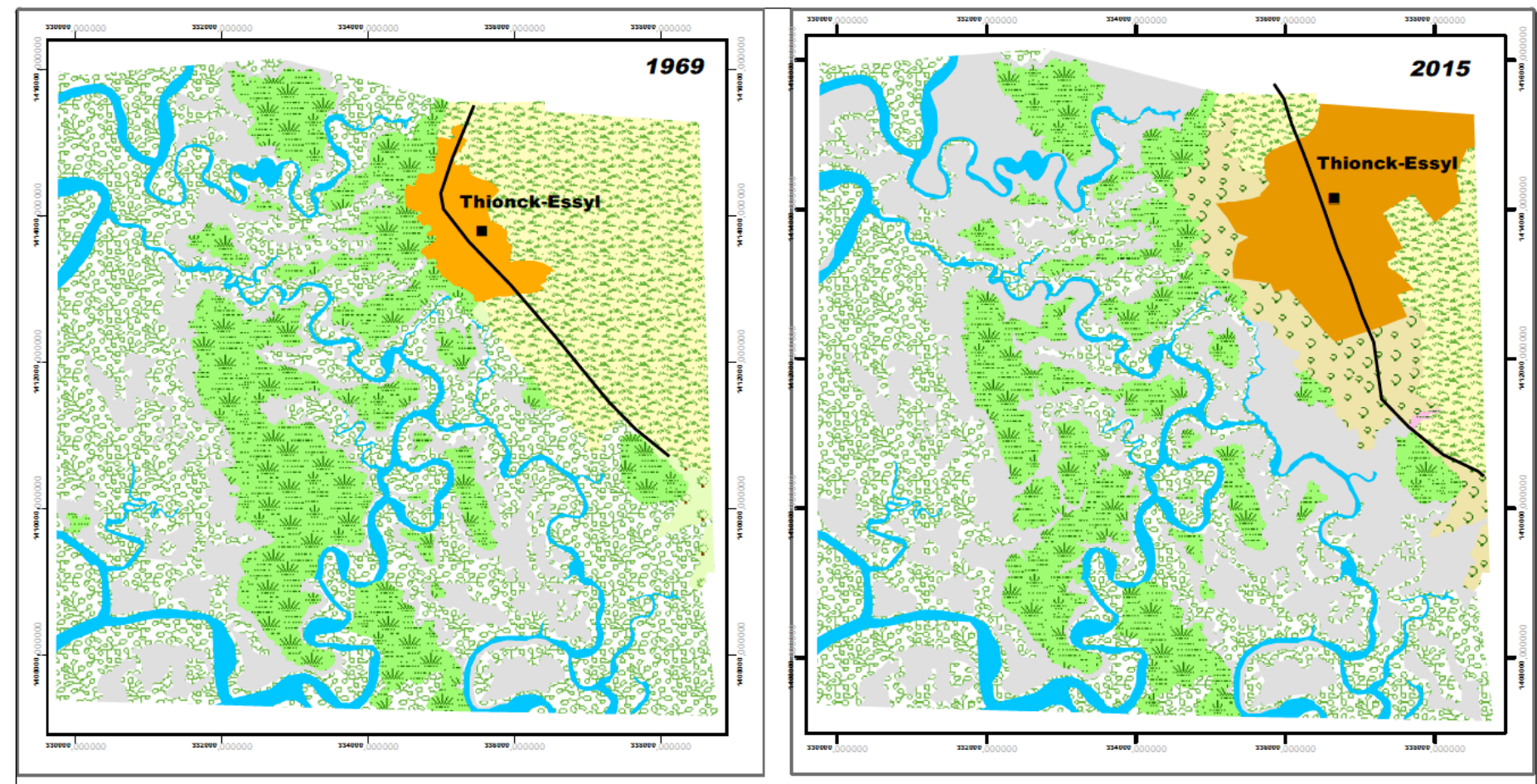

- Localité
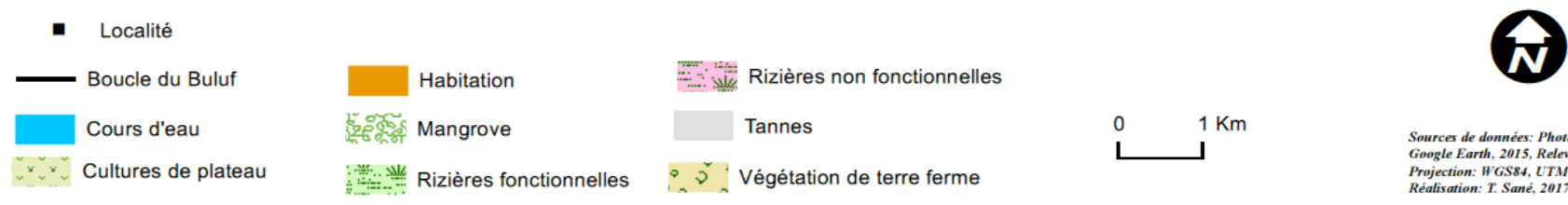

Sources de données: Photo aérienne 1969.
Google Earth, 2015, Relevés de terrain Projection: WGS84, UTM, Zone 28N

Figure 9 : Dynamique des unités paysagères du terroir rizicole villageois de Thionck-Essyl entre 1969 et en 2015

A Coubanao, la perte des terres rizicoles est encore plus importante qu'à Thionck-Essyl puisque leur recul est chiffré à 38,9\% (tabl. 4). La régression des parcelles rizicoles ainsi que celle de la mangrove se confirme à travers l'extension des tannes $(+69,4 \%)$. La figure 10 rend compte de la spatialisation des différents faciès du paysage rizicole du terroir villageois.

Tableau 4 : Evolution des statistiques d'occupation des sols à Coubanao entre 1969 et 2015

\begin{tabular}{|l|c|c|c|c|c|}
\hline Unités paysagères & $\begin{array}{c}\text { Superficies } \\
\text { en 1969 } \\
\text { (ha) }\end{array}$ & $\begin{array}{c}\text { Superficies } \\
\text { en \% }\end{array}$ & $\begin{array}{c}\text { Superficies } \\
\text { en 2015 } \\
\text { (ha) }\end{array}$ & $\begin{array}{c}\text { Superficies } \\
\text { en \% }\end{array}$ & $\begin{array}{c}\text { Progression/régression } \\
\text { (en \%) }\end{array}$ \\
\hline Cultures de plateau & 209,9 & 16,7 & 88,3 & 7 & $-57,9$ \\
\hline Habitation & 57,7 & 4,6 & 145,3 & 11,6 & 151,8 \\
\hline Mangrove & 117,8 & 9,4 & 25,8 & 2,1 & $-78,1$ \\
\hline Rizières & 534,3 & 42,6 & 326,4 & 26 & $-38,9$ \\
\hline $\begin{array}{l}\text { Rizières } \\
\text { fonctionnelles }\end{array}$ & - & - & 106,3 & 8,5 & 8,5 \\
\hline Tannes non & 245,9 & 19,6 & 416,5 & 33,2 & 69,4 \\
\hline $\begin{array}{l}\text { Végétation terre } \\
\text { ferme }\end{array}$ & 89,3 & 7,1 & 146,3 & 11,7 & 63,8 \\
\hline Total & $\mathbf{1 2 5 4 , 8}$ & $\mathbf{1 0 0}$ & $\mathbf{1 2 5 4 , 8}$ & $\mathbf{1 0 0}$ & - \\
\hline
\end{tabular}




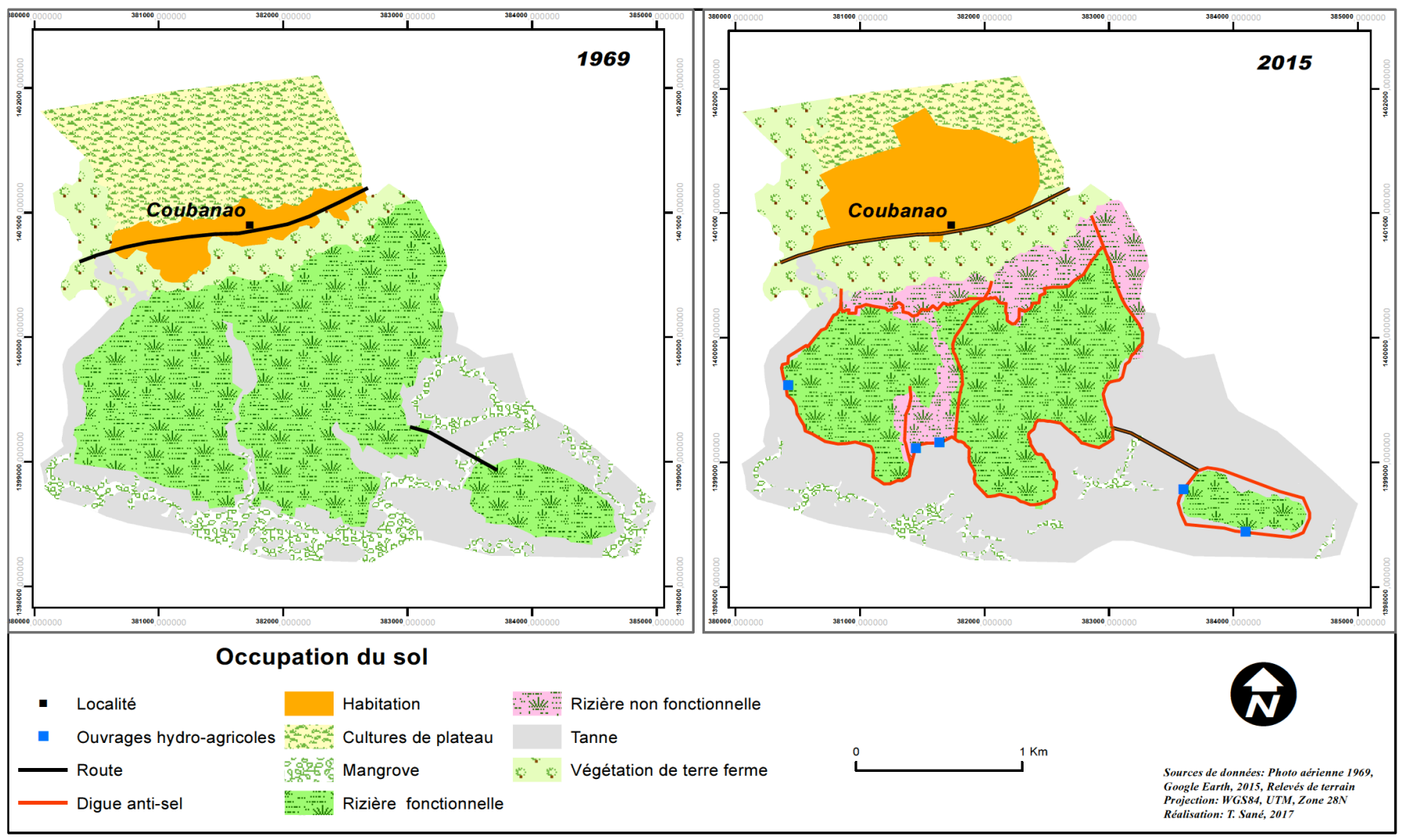

Figure 10 : Dynamique des unités paysagères du terroir rizicole villageois de Coubanao en 1969 et en 2015

Les dynamiques observées à Thionck-Essyl et à Coubanao sont relativement différentes de celles qui se manifestent à Diembering. Ici, la perte de terres rizicoles est moins importante. En effet, en plus des rizières abandonnées $(0,9 \%)$, le recul des terres rizicultivables est de l'ordre de 10,5\% entre 1969 et 2015. La régression de la mangrove est ici faible, $-0,5 \%$. Seuls ces deux faciès ont connu un recul de leurs superficies. Toutes les autres unités paysagères ont connu une progression de leurs surfaces (tabl. 5). La figure 11 traduit l'occupation spatiale des différentes unités paysagères à Diembering en 1969 et en 2015.

Tableau 5 : Evolution des statistiques d'occupation des sols à Diembering entre 1969 et 2014

\begin{tabular}{|l|l|l|l|l|c|}
\hline \multicolumn{1}{|c|}{ Unités paysagères } & $\begin{array}{c}\text { Superficies } \\
\text { en 1969 (ha) }\end{array}$ & $\begin{array}{c}\text { Superficies } \\
\text { en \% }\end{array}$ & $\begin{array}{c}\text { Superficies en } \\
\mathbf{2 0 1 5}(\mathbf{h a})\end{array}$ & $\begin{array}{c}\text { Superficies } \\
\text { en \% }\end{array}$ & $\begin{array}{c}\text { Progression/régression } \\
\text { (en \%) }\end{array}$ \\
\hline Cours d'eau & 416,6 & 5,7 & 423,9 & 5,8 & 1,8 \\
\hline Dunes & 1,7 & 0 & 31,6 & 0,4 & 1758,8 \\
\hline Habitation & 52,6 & 0,7 & 58,5 & 0,8 & 11,2 \\
\hline Mangrove & 1945 & 26,8 & 1934,5 & 26,7 & $-0,5$ \\
\hline Océan & 742,5 & 10,2 & 755,4 & 10,4 & 1,7 \\
\hline Plage & 178,6 & 2,5 & 125,7 & 1,7 & $-29,6$ \\
\hline Rizières & 2254,5 & 31,1 & 2017,6 & 27,8 & $-10,5$ \\
\hline Rizières non fonctionnelles & & & 62 & 0,9 & 0,9 \\
\hline Tannes & 126,3 & 1,7 & 155,3 & 2,1 & 23,0 \\
\hline Végétation terre ferme & 1362,9 & 18,8 & 1499,5 & 20,7 & 10,0 \\
\hline Cultures de plateau & 173,1 & 2,4 & 171,4 & 2,4 & $-1,0$ \\
\hline Plantes typha & - & - & 18,3 & 0,3 & 0,3 \\
\hline Total & $\mathbf{7 2 5 3 , 8}$ & $\mathbf{1 0 0}$ & $\mathbf{7 2 5 3 , 8}$ & $\mathbf{1 0 0}$ & - \\
\hline
\end{tabular}




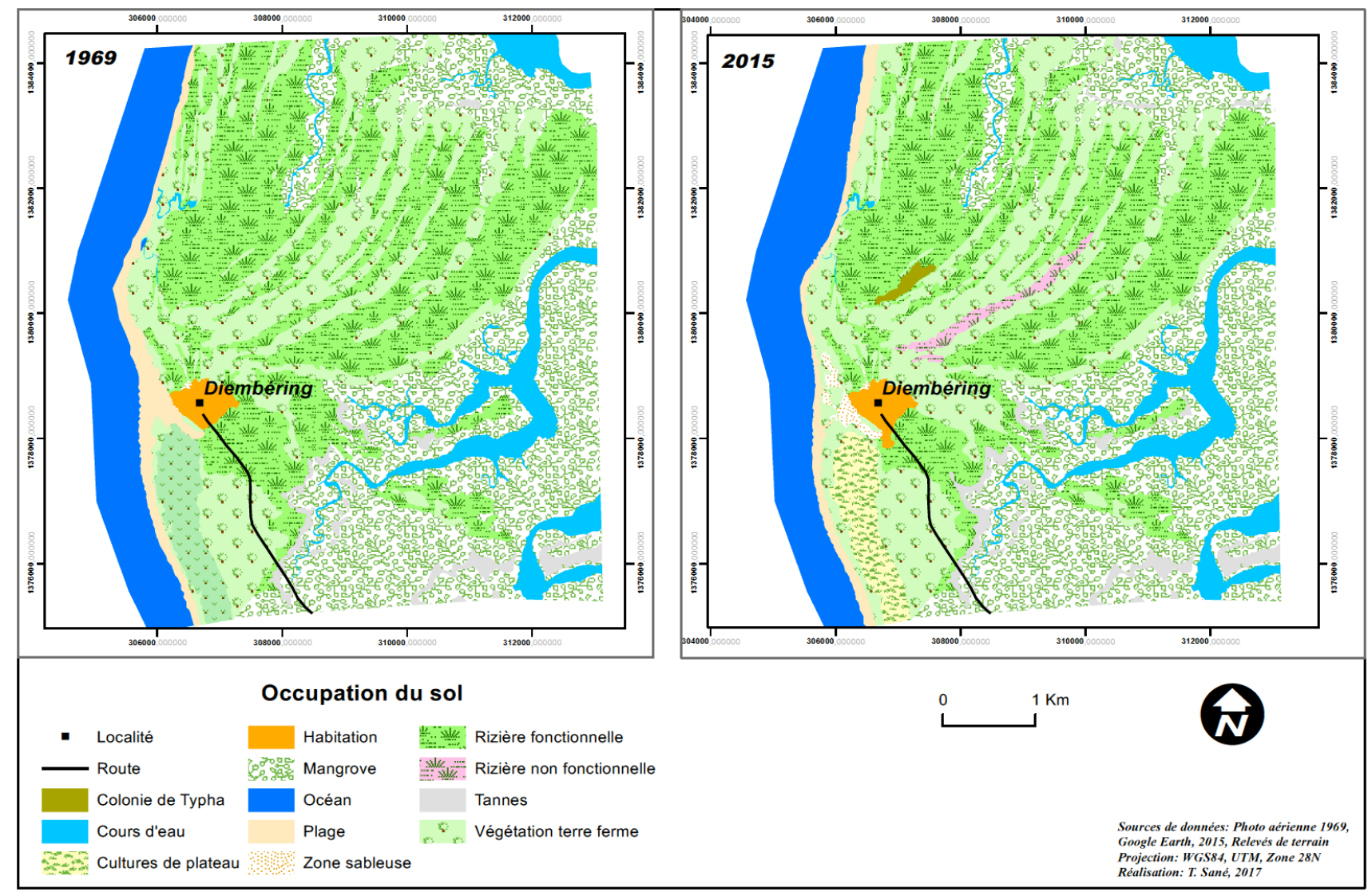

Figure 11 : Dynamique des unités paysagères du terroir rizicole villageois de Diembering entre 1969 et en 2015

\section{IV - Les politiques publiques agricoles : une des réponses aux difficultés de la riziculture en Basse-Casamance}

Les déficits pluviométriques et la dégradation de l'environnement biophysique sont, pour l'essentiel, à l'origine des politiques publiques d'aménagement rural en Basse Casamance. L'analyse des politiques publiques de développement agricole dans cette zone révèle que la volonté exprimée par les autorités sénégalaises d'aménager les vallées rizicoles de la région remonte aux années 1960, période où la production arachidière était considérée comme une priorité pour le pays. Il s'agissait, à cette époque d'accroître les surfaces rizicoles et d'améliorer leur productivité, avec comme objectif d'assurer la sécurité alimentaire en riz du pays. Cette volonté s'est concrétisée par la mise en place de plusieurs programmes et projets de développement agricole successivement réalisés en Basse-Casamance. Durant les deux premières décennies de l'indépendance du pays, les politiques publiques agricoles furent d'abord mises en œuvre par les structures étatiques destinées à contrôler les conditions socioéconomiques de la production. C'est ainsi que la priorité fut accordée à la création de structures administratives nationales chargées de promouvoir ou d'infléchir le développement agricole. Dans cette logique, sont nés des organismes étatiques. Il s'agit, entre autres, de la Société de Mise en Valeur Agricole de la Casamance (SOMIVAC) en Basse-Casamance et la Société de Développement et de Vulgarisation Agricole (SODEVA) dans le bassin arachidier. Ces sociétés sont chargées d'assurer plus ou moins directement la recherche agronomique, la formation des paysans, la mise en place d'infrastructures, l'offre de crédits et de subventions, la production et la certification de semences, l'approvisionnement en intrants, l'assistance technique, les soins vétérinaires, la commercialisation des produits agricoles, la stabilisation des prix, les redistributions foncières (Dufumier, 1993). En BasseCasamance, les difficultés rencontrées sur le terrain après une expérience de plus de deux décennies d'aménagements et l'échec retentissant des projets réalisés (ILACO, barrages de Guidel et d'Affiniam) ont sans aucun doute suscité la remise en question du modèle de développement agricole retenu par l'Etat du Sénégal dans le contexte des plans d'ajustement structurels. L'erreur de ces plans a été de 
concevoir la force de travail comme un simple "facteur de production" à l'égal des autres ressources disponibles (terre, équipements, capital circulant, etc.), sans prendre en compte les intérêts des d'agriculteurs. Devant l'ampleur des dégâts occasionnés sur le milieu et les échecs répétés, l'Etat a voulu corriger ces erreurs en démarrant en 1984 un programme de construction de petits barrages ou digues anti-sel, sous l'égide du Projet Intégré de Développement Agricole en Casamance(PIDAC) en collaboration avec les villageois, qui ont été les initiateurs du projet à l'échelle locale. Leur participation aux travaux, à la maintenance et à la gestion des ouvrages réalisés a semblé être un gage de succès. Malheureusement, le conflit armé dont le début remonte à l'année 1982 mettra en péril les résultats escomptés. La dégradation des conditions climatiques et les effets du conflit vont se conjuguer et se traduire par la baisse des revenus des producteurs ruraux, désorganisant ainsi les forces productives et les conditions de la production agricole. De la recherche d'une paix durable dans la région, vont naître d'autres programmes et projets de développement comme le Projet d'Appui au Développement Rural de la Casamance(PADERCA) dans le souci de soutenir l'effort de relance des activités socioéconomiques à travers l'Agence Nationale pour la Relance des Activités économiques et sociales en Casamance (ANRAC). Il faut également mentionner le rôle très important que les organisations non gouvernementales (ONG) comme le Groupe de recherche et de réalisations pour le développement rural (Grdr) et autres partenaires œuvrant pour le développement rural ont joué, au cours de ces dernières années, dans un contexte de conflit armé, dans le maintien des activités rizicoles à travers différentes initiatives de protection et de réhabilitation des systèmes productifs dégradés et d'accompagnement des organisations paysannes.

Ces différents projets ont connu des fortunes diverses et n'ont pas pu réellement apporter des éléments de réponse adéquate par rapport aux attentes de la population paysanne. Parmi les facteurs de l'échec des politiques agricoles, on peut noter le manque de coordination des interventions entre publics, ONG et privés, l'absence d'industrie agro-alimentaire et de régulation du marché, et le manque d'inscription dans la durée des actions menées sur le terrain, avec une logique de contrat, préjudiciable à la durabilité des interventions.

\section{V - Les stratégies récentes de diversification des revenus}

Dans un contexte de contraintes parfois difficiles à surmonter, le riz local, longtemps nourriture de base et fondement du système agraire en Basse-Casamance, est peu à peu marginalisé au profit de ressources plus diversifiées et d'importation du riz brisé asiatique (même le riz casamançais est désormais brisé) qui demeure très importante au Sénégal (fig. 12). En effet, l'importation du riz (y compris la contrebande en provenance surtout de la Gambie), devenue une nécessité à cause des multiples contraintes analysées ici et la faible productivité de la riziculture traditionnelle, met en cause la souveraineté alimentaire et révèle les limites de la résilience de la société diola dans un contexte de diminution quasi-générale des superficies rizicoles dans la zone au cours des dernières. 


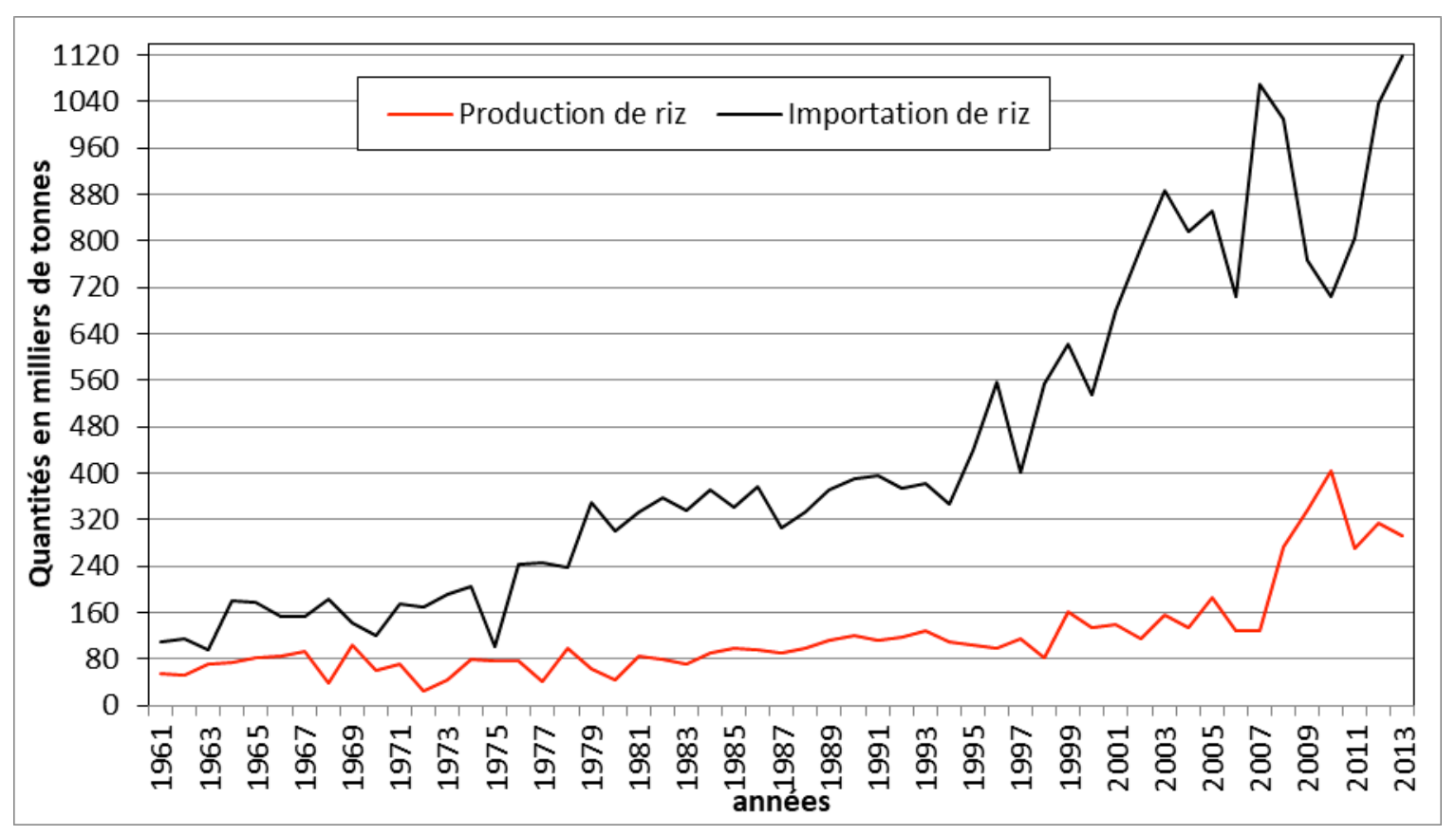

Figure 12 : Importation versus production de riz au Sénégal (source : FAO stat, 2016, Sané, 2017)

Il faut également souligner que le développement des stratégies de diversification des revenus (Grdr et al., 2017) peut être considéré comme une réponse au recul des terres rizicultivables et à la stérilisation de certains sols par le sel, l'acidification et l'ensablement. Dans tous les secteurs d'activités, les éléments de vitalité relative de la diversification des sources de revenus en Basse-Casamance sont notés. Il s'agit, entre autres, de l'engagement des Diola, depuis quelques décennies dans la pêche maritime et la revalorisation d'anciens usages de la mangrove liée à l'amélioration des conditions environnementales depuis la fin des années 90 ; la redynamisation des activités touristiques le long du littoral de la Basse-Casamance, avec d'importantes recompositions dans les terroirs des principales stations touristiques comme Cap-Skirring et Kafountine (Ehemba et al., 2017; Sy et al., 2018). L'extraversion économique (Marut, 2016), l'exploitation forestière de plus en plus importante et le développement des vergers (mangues et agrumes) et autres plantations d'anacardiers, surtout dans la partie sud et sud-est frontalière à la Guinée-Bissau, constituent aussi des éléments importants de la recomposition économique des terroirs de Basse-Casamance. Il faut cependant souligner que la monoplantation d'anacardiers dans la plupart des zones (risque d'épuisement des sols) et le peu de valorisation sur place des produits qui en sont issus, sont des facteurs limitants non négligeables pour cette filière. La vente des terres dans les zones touristiques, surtout pour les résidences secondaires au Cap-Skirring et dans l'axe Kafountine-Abéné, participe à la remise en cause des structures traditionnelles de gestion foncière (Sané, 2017 ; Ehemba et al., 2017 ; Sy et al., 2018).

\section{Conclusion}

$\mathrm{Au}$ terme de notre analyse sur les dynamiques des terroirs rizicoles de Basse-Casamance, on peut retenir les tendances majeures suivantes :

- Une pluviométrie à variabilité relativement forte et globalement très instable qui contraint les paysans d'anticiper / gérer les risques ;

- un environnement biophysique en mutation caractérisé par la remontée des eaux marines dans tous les marigots du réseau hydrographique du fleuve Casamance avec comme conséquence la salinité des eaux et des terres rizicoles; 
- la difficile mobilisation de la main d'œuvre, induite à la fois par la baisse de la productivité des terres, la pénibilité des travaux rizicoles et le manque d'opportunités réelles pour les jeunes en milieu rural ;

- le recul quasi-généralisé des terres rizicultivables dont certaines sont faiblement ou non entretenues et envahies par les eaux salées devenant moins propices à la riziculture ;

- si les politiques publiques agricoles et d'aménagements hydrauliques n'ont pas résolu les problèmes auxquels la Basse-Casamance a été et reste confrontée, l'essentiel du fondement social, le riz, est préservé comme en témoignent les chiffres de superficies que cette culture occupe encore dans les trois terroirs-témoins.

Dans le contexte actuel de recomposition des terroirs rizicoles liée aux changements globaux et aux pratiques agricoles peu adaptées, les politiques publiques rizicoles et les interventions des ONG (aménagements hydro-agricoles, projets et programmes de développement rural) ne semblent pas pour le moment donner des résultats escomptés. Néanmoins, il est remarquable de constater la capacité d'adaptation des systèmes ruraux. Les communautés rurales tentent de répondre à ces changements en modifiant certaines composantes de leurs systèmes d'exploitation. La recomposition des combinaisons "ressources-techniques-acteurs" a été rendue possible précisément du fait des multiples usages des ressources naturelles. Par ailleurs, la maîtrise poussée et ingénieuse des ressources des espaces littoraux et maritimes par les communautés littorales a été maintenue moyennant un certain nombre d'innovations (techniques, sociales, économiques, institutionnelles). Il s'agit, entre autres, de la revalorisation d'anciens usages de la mangrove (récolte du sel, des huîtres, des coquillages, du bois, migrations maritimes en matière de pêche) et d'autres activités (arboriculture, plantation d'anacardiers, etc.) dans des contextes politiques et économiques particuliers.

\section{Références bibliographiques}

Barbier-Wiesser F. G., 1994 (dir.) : comprendre la Casamance. Chronique d'une intégration contrastée. Paris, Editions Karthala, 507p.

Bezançon G., 1994 : Le riz cultivé d'origine africaine Oryza glaberrima Steud et les formes sauvages et adventices apparentées : diversité, relations génétiques et domestication. Editions Orstom, Collection Travaux et Documents Microédités, 249p.

Boivin P., 1991 : Caractérisation physique des sols sulfatés acides de la vallée de Katouré (Basse Casamance, Sénégal. Etude de la variabilité spatiale et relation avec les caractéristiques pédologiques. Editions ORSTOM, Institut Français de Recherche Scientifique pour le Développement en Coopération, Collection ÉTUDES et THÈSES, 232p.

Bonnefond Ph., Loquay A., 1985 : Aspects socio-économiques de la riziculture en Basse et Moyenne

Casamance. Paris, République française, Ministère des relations extérieures, coopération et développement, 428p.

Chéneau-Loquay A., 1994a: "La raison. Géographie "des" Casamance". In Comprendre la Casamance, chronique d'une intégration contrastée. Barbier-Wiesser (ed.), Paris, Karthala, coll. Hommes et Sociétés, pp. 47-68.

Chéneau-Loquay A., 1994b : "Demain, encore le riz? Fin d'une civilisation ?" In Comprendre la Casamance, chronique d'une intégration contrastée. Barbier-Wiesser (ed.), Paris, Karthala, coll. Hommes et Sociétés, pp. 351-383.

Cormier-Salem, M. C., 1985 :"Les jeunes Diola face à l'exode rural". Cahiers ORSTOM, série Sciences humaines, 21 (2-3), pp. 267-273.

Cormier-Salem, M. C., 1992 : Gestion et évolution des espaces aquatiques : La Casamance. Paris, Editions de l'ORSTOM, Collection Etudes et Thèses, 583p. 
Cormier-Salem, M. C., 1994 (ed. sc.) : Dynamique et usages de la mangrove dans les pays des rivières du sud (du Sénégal à la Sierra-Léone. ORSTOM Éditions, Collection Colloques et Séminaires, 357p.

Cormier-Salem, M. C., 1995 : "Du riz, des poissons, des hommes. Stratégies paysannes des populations littorales de Rivières du Sud (du Sénégal à la Sierra Leone)". Colloque international, "Quel avenir pour les rizicultures de l'Afrique de l'Ouest",CNRS/CIRAD, Bordeaux, 4-7 avril 1995.

Cormier-Salem, M. C., 1999 (ed.) : Rivières du Sud. Sociétés et mangroves ouest-africaines. Editions de l'IRD, vol. 1, 426p.

Cormier-Salem M., Sané T., 2017: Définir un cadre méthodologique commun en cartographie participative. Revue d'ethnoécologie [En ligne], 11 | 2017, mis en ligne le 03 juillet 2017, URL : http://ethnoecologie.revues.org/2930 ; DOI : 10.4000/ ethnoecologie.2930.

Dacosta H., 1989 : Précipitations et écoulements sur le bassin de la Casamance. Thèse $3^{\text {ème }}$ cycle, université Cheikh Anta Diop, Dakar, 283 p.

Descroix L., Djiba S., Sané T., Tarchiani V. (eds), 2015 : Eaux et sociétés face au changement climatique dans le bassin de la Basse Casamance. Paris, L'Harmattan, 240p.

Diédhiou L., 2001 : Projets de développement et représentations sociales en Basse Casamance : le $D E R B A C$ et le PROGES. Thèse de Doctorat, Université de Montréal, Canada, 425p.

Diop A., 2017 : Evolution de l'occupation des sols et de la main d'œuvre rizicole dans le Bandial en Basse Casamance. Mémoire de Master de Géographie, Département de Géographie, Université Assane Seck de Ziguinchor, 103p.

Diop E. H. S., 1990 : La côte ouest-africaine. Du Saloum (Sénégal) à la Mellacorée (République de Guinée). Editions ORSTOM, Institut Français de Recherche Scientifique pour le Développement en Coopération, Collection ÉTUDES et THÈSES, 366p.

Dufumier M., 1993 : Politiques agricoles et initiatives locales. In "Dynamiques des systèmes agraires : politiques agricoles et initiatives locales, adversaires ou partenaires". ORSTOM-Editions, Colloques et Séminaires, pp. 15-47.

Ehemba F., Lecoq Y., Ruë O., Niokane M., Bodivit M., Seck P., Diallo M., Agossou S., Dexcroix L., Sané T., Fabre M., 2017 : "Une cartographie participative pour bâtir une connaissance concertée des mutations de territoire dans l'espace transfrontalier sénégalo-bissao-guinéen". Actes du colloque international du Laboratoire Mixte International "Patrimoines et Territoires de l'Eau", du 11 au 14 mai 2016, Université Gaston Berger de Saint-Louis du Sénégal, pp. 241-263.

Fall A. S., Guèye C., Tall S. M., 2005 : "Changements climatiques, mutations urbaines et stratégies citadines à Dakar (Sénégal)". In Urbain-Rural : l'hybridation en marche, Enda Tiers-Monde, p : 190231.

Grdr, Sané T., Dieye E. B, Descroix L., 2017. Un littoral en mouvement : Diversité, dynamiques et mutations des territoires frontaliers du sud-ouest du Sénégal et du nord-ouest de la Guinée-Bissau (Ö̈o, Cacheu, Ziguinchor, Sédhiou), juin 2017, 140p.

Guèye M., - Sivakumar M.V.K., 1992 : Analyse de la longueur de la saison culturale en fonction de la date de début des pluies au Sénégal. Centre Sahélien de l'ICRISAT, Compte Rendu des Travaux $n^{\circ} 2$, Niamey, Niger, $42 \mathrm{p}$.

Gourou P., 1984 : Riz et civilisation.Paris Fayard, 229p.

Harza, 1981: "Master Plan of agricultural development of the Lower Casamance". S.1., Harza International/SOMIVAC, Area Interim Report, 15-09- 1981. 
Linares 0. De Sapir, 1970:"Agriculture and diola society". In Mc.Lgughlin P.F. (ed.), African Food Production Systems: Cases and theory.Baltimore and London, The John Hopkins Press, pp. 195-227.

Loquay A., 1979 : Thionck-Essyl en Basse Casamance : évolution récente de la gestion des ressources renouvelables. Thèse de doctorat de $3^{\text {ème }}$ cycle, Université Bordeaux III, $407 \mathrm{p}$.

Marius C., 1985 : Mangroves du Sénégal et de la Gambie. Écologie, Pédologie, Géochimie, Mise en valeur et aménagement. Travaux et Documents de l'ORSTOM, Paris, 193, 368p.

Marut J. C., 2016 : "Changement climatique, paix et développement : des choix citoyens". L'Harmattan, Actes de l'atelier scientifique et du lancement de l'initiative Casamance : un réseau scientifique au service du développement en Casamance, Ziguinchor, Sénégal, pp.28-42.

Marzouk Y., 1980 : "Socio Economic Study of Agriculture in Lower Casamance". Paper Presented for the Workshop on "Sahelian Agriculture", Department of Agricultural Economics, Purdue University, pp. 117-153.

Marzouk Y., 1991: "Histoire des conceptions hydrauliques étatiques et paysannes en BasseCasamance, Sénégal, 1960-1990". In Savoirs paysans et développement, Paris, Karthala-ORSTOM, pp.61-97.

Montoroi J. P., 1996 : Gestion durable des sols de la mangrove au Sénégal en période de sécheresse. Dynamique de l'eau et géochimie des sels d'un bassin versant aménagé. Editions ORSTOM, Institut Français de Recherche Scientifique pour le Développement en Coopération, Collection Études et Thèses, 266p.

Mougenot B., Zante P., Montoroi J. P., 1990 : "Détection et évolution saisonnière des sols salés et acidifiés du domaine fluvio-marin de basse Casamance au Sénégal, par imagerie satellitaire". John Libbey Eurotex, Revue Télédétection et sécheresse, Éditions AUPELF-UREF, pp.173-179.

Portères R., 1952: "Les Rizières de ruissellement en Casamance". In : Revue internationale de botanique appliquée et d'agriculture tropicale, 32 ${ }^{\mathrm{e}}$ année, bulletin $\mathrm{n}^{\circ} 351-352$, Janvier-février 1952. pp.34-37. http://www.persee.fr/doc/jatba_0370-5412_1952_num_32_351_6769.

Pélissier P., 1958 : Les Diola : Etude sur l'habitat des riziculteurs de Basse Casamance. Université de Dakar, Département de géographie, Faculté des lettres, 65p.

Pélissier P., 1966 : Les paysans du Sénégal : les civilisations agraires du Cayor à la Casamance. Paris, imprimerie Saint-Yrieix, 944p.

Sané T., 2017 : Vulnérabilité et adaptabilité des systèmes agraires à la variabilité climatique et aux changements sociaux en Basse-Casamance (Sud-Ouest du Sénégal). Thèse de Doctorat unique, Université Paris Diderot - Paris 7, 376p.

Sané T., Cormier-Salem M. C., Dieye E. B., Descroix L., Fabre M., Habert E., Ehemba F., Bodivit M., Sy O., Ba B. D., Mendy V., 2017 : "La cartographie participative comme outil d'aide à la compréhension des dynamiques territoriales : application sur un terrain de Basse-Casamance (Sénégal)". Actes du colloque international du Laboratoire Mixte International "Patrimoines et Territoires de 1'Eau", du 11 au 14 mai 2016, Université Gaston Berger de Saint-Louis du Sénégal, pp. 265-287.

Sané T., Sy O., Dieye E. B., Descroix L., Diaw A. T., 2015 : "De la pertinence des grands aménagements hydro-agricoles dans un contexte d'instabilité climatique : cas du barrage d'Affiniam en Basse Casamance". In "Eaux et Sociétés face au changement climatique dans le bassin de la Casamance". Actes de l'atelier scientifique et du lancement de l'initiative Casamance : un réseau scientifique au service du développement. L'Harmattan, pp.117-132. 
Sané T., Diop M., Sagna P., 2008 : "Etude la qualité de la saison pluvieuse en Haute-Casamance (Sud Sénégal)". In Sécheresse vol. 19 n¹, janvier-février-mars 2008, pp. 1-6.

Sy O., Sané T., 2008 : "Changements climatiques et crise de la riziculture en Basse-Casamance (Sénégal)". In "Climats et risques climatiques en Méditerranée", Actes du XXIème colloque de l'Association Internationale de Climatologie (AIC), 9-13 septembre 2008 à Montpellier (France), pp.587-591.

Sy O., Sané T., Dieye E. B., 2018 : "Dynamique et aménagement de la frange littorale de la commune rurbaine de Diembering au Sénégal". In Rubén C. Lois-González Yamilé Pérez-Guilarte Rosa Verdugo-Matés, Afrique de l'Ouest: Questions sur le développement à l'école locale, pp. 197-221.

Thomas, L. V., 1959 : Les Diola. Essai d'analyse fonctionnelle sur une population de BasseCasamance. Dakar, Mémoires IFAN, 55 (Thèse d'Etat publiée, 2 vol.), 821 p.

Thomas L.V., 1960a : "L'organisation foncière des Diola (Basse Casamance)". Annales Africaines, 1, pp. $199 \cdot 223$.

Thomas L.V., 1960b : "Esquisse sur les mouvements de population et les contacts socio-culturels en pays diola (Basse Casamance)". Dakar, Bulletin de l'IFAN, série B. Sciences humaines, vol.22, pp.486-508. 